\title{
Global Regularity for Vortex Patches ${ }^{\star \star}$
}

\author{
A. L. Bertozzi ${ }^{\star}$ and P. Constantin ${ }^{\star \star}$ \\ Department of Mathematics, University of Chicago, Chicago, IL 60637, USA
}

Received March 24, 1992; in revised form August 3, 1992

Abstract. We present a proof of Chemin's [4] result which states that the boundary of a vortex patch remains smooth for all time if it is initially smooth.

\section{Introduction}

A vortex patch is a domain $D$ (simply connected, open and bounded) in the Euclidean plane $\mathbf{R}^{2}$ which moves with a velocity given at each instant of time by

with

$$
v(x)=\nabla^{\perp} \Psi(x)=\left(\begin{array}{c}
-\frac{\partial \Psi}{\partial x_{2}} \\
\frac{\partial \Psi}{\partial x_{1}}
\end{array}\right)
$$

$$
\Psi(x)=\frac{\omega_{0}}{2 \pi} \int_{D} \ln |x-y| d y,
$$

where the constant $\omega_{0}$ is time independent. Vortex patches are particular examples of weak solutions of the two dimensional incompressible Euler equation. Wellknown results of Yudovich [10] provide a framework for the vortex patch problem. If an initial domain $D_{0}$ and a constant $\omega_{0}$ are prescribed then there exists a unique vortex patch $D(t)$, defined for all time $t \in \mathbf{R}$ which starts out at $t=0$ from $D_{0}$ : $D(0)=D_{0}$. The area of $D(t)$ is constant in time but, in general, other geometric features behave in a less regular fashion. In particular, the length or curvature of the boundary may grow rapidly $[1,6]$. If the boundary is smooth enough $\left(C^{1}\right)$, then the velocity is a contour integral on the boundary, and so the problem can be expressed as a self deforming curve in the plane [11] (the curve is the patch

\footnotetext{
* Partially supported by a National Science Foundation Postdoctoral Fellowship

** Partially supported by the National Science Foundation
} 
boundary). Majda [8], proposed the vortex patch problem, in contour dynamic form, as a model for the inviscid, incompressible creation of small scales. Motivated by analogy with the stretching of vorticity in three dimensions and by a simple model [5] he suggested the possibility of finite time singularities. In other words, some smooth initial contours might, in finite time, lead to loss of regularity (infinite length, corners, or cusps for instance). This suggestion has been the subject of some debate in the computational literature $[3,7]$.

Recently, Chemin [4] proved that smooth contours stay smooth for all time. We offer here our interpretation of Chemin's result.

The absence of blow up is mainly due to kinematic ("frozen time") rather than time dependent reasons. We use the word "kinematic" somewhat abusively to refer to spatial properties of the velocity deduced from the Biot-Savart law. In order to explain them let us compute $\nabla v$ :

$$
\nabla v(x)=\frac{\omega_{0}}{2 \pi} P_{v} \int_{D} \frac{\sigma(x-y)}{|x-y|^{2}} d y+\frac{\omega_{0}}{2}\left(\begin{array}{rr}
0 & -1 \\
1 & 0
\end{array}\right) \chi_{D}(x) .
$$

Here the $P v$ stands for Cauchy's principal value, $\chi_{D}(x)$ equals 1 in $D, \frac{1}{2}$ on $\partial D$ and 0 outside $\bar{D}$. The $2 \times 2$ symmetric matrix $\sigma(z)$ is explicit

$$
\sigma(x)=\frac{1}{|z|^{2}}\left(\begin{array}{cc}
2 z_{1} z_{2} & z_{2}^{2}-z_{1}^{2} \\
z_{2}^{2}-z_{1}^{2} & -2 z_{1} z_{2}
\end{array}\right)
$$

but what is important is that

- it is a smooth function, homogeneous of degree 0 ,

- it has zero mean on the unit circle, and

- it is symmetric with respect to reflections

$$
\sigma(-z)=\sigma(z) .
$$

It is obvious from formula (1.3) that the gradient of $v$ is discontinuous across the boundary of $D$, no matter how smooth this boundary might be.

The first kinematic observation is that $|\nabla v(x)|$ is bounded across smooth $\left(C^{1, \mu}\right)$ boundaries. This happens because near the boundary of $D$ the intersection of $D$ with a small circle looks very much like a half-circle (Geometric Lemma) and because, in view of (1.5) the mean of $\sigma$ on half-circles is zero. Not unexpectedly the bound turns out to be logarithmic,

$$
|\nabla v|_{L^{\infty}} \leqq C_{\mu}\left|\omega_{0}\right|\left(1+\left(\log \left[\Delta_{\mu}\right]\right)\right),
$$

where $\Delta_{\mu}$ is related to the $\mu$-Hölder modulus of continuity of the tangent to the boundary (Proposition 1).

The second kinematic observation is that despite its discontinuity, $\nabla v$ is nevertheless continuous in the tangential direction. More precisely the formula (Proposition 2)

$$
\nabla v(x) \mathbf{W}=\frac{\omega_{0}}{2 \pi} P_{v} \int_{D} \frac{\sigma(x-y)}{|x-y|^{2}}(\mathbf{W}(x)-\mathbf{W}(y)) d y
$$

holds for divergence free vector fields $\mathbf{W}$ which are tangent to $\partial D$. Such a vector field is

$$
\mathbf{W}=\nabla^{\perp} \varphi
$$


for $\varphi$ a $C^{1, \mu}$ function in $\mathbf{R}^{2}$ which defines the patch via

$$
D=\left\{x \in \mathbf{R}^{2} \mid \varphi(x)>0\right\} .
$$

From the formula (1.7) it follows by classical arguments that the $\mu$-Hölder modulus of continuity of $(\nabla v) \nabla^{\perp} \varphi$ is bounded by the product of $|\nabla v|_{L^{\infty}}$ and the $\mu$-Hölder modulus of continuity of $\nabla^{\perp} \varphi$. In view of the first kinematic observation concerning $|\nabla v|_{L^{\infty}}(1.6)$ we deduce the bound

$$
\left|(\nabla v) \nabla^{\perp} \varphi\right|_{\mu} \leqq C_{\mu}\left|\omega_{0}\right|\left(1+\left(\log \left[\Delta_{\mu}\right]\right)\right)\left|\nabla^{\perp} \varphi\right|_{\mu}
$$

which is only logarithmically superlinear. The quantity $(\nabla v) \nabla^{\perp} \varphi$ is the material derivative of $\nabla^{\perp} \varphi$ if $\varphi$ is transported passively, i.e.

and

$$
\left(\frac{\partial}{\partial t}+v \cdot \nabla\right) \varphi=0
$$

$$
\left(\frac{\partial}{\partial t}+v \cdot \nabla\right) \nabla^{\perp} \varphi=\nabla v \nabla^{\perp} \varphi .
$$

It is only here that the time dependence enters at the level of a Gronwall inequality (Proposition 3) and provides an a priori bound for $|\nabla \varphi|_{\mu}$, the $\mu$-Hölder modulus of continuity of the tangent. This however is known to be sufficient for any higher derivatives, by classical calculus inequalities (see for example [2]). The conclusion is that $|\nabla v|_{L^{\infty}}$ may grow like $e^{C t}$ and quantities related to smoothness of the boundary (such as length, curvature, etc.) may grow like $e^{c_{1} e^{c_{2} t}}$.

\section{The Main Result}

We reformulate the vortex patch problem as follows. We seek a scalar $\varphi(x, t)$ which solves

$$
\begin{aligned}
\frac{\partial \varphi}{\partial t}+v \cdot \nabla \varphi & =0, \\
\varphi(x, 0) & =\varphi_{0}(x),
\end{aligned}
$$

where $v$ is given by the Biot-Savart law

$$
v(x, t)=\frac{\omega_{0}}{2 \pi} \int_{D} \nabla_{x}^{\perp} \ln |x-y| d y,
$$

and $D=D(t)$ is given by

$$
D=\left\{x \in \mathbf{R}^{2} \mid \varphi(x)>0\right\} .
$$

We assume that

$$
D_{0}=\left\{x \in \mathbf{R}^{2} \mid \varphi_{0}(x)>0\right\}
$$

is bounded and has a smooth boundary. We also assume that

$$
\inf _{x \in \partial D_{0}}\left|\nabla \varphi_{0}(x)\right| \geqq m>0
$$


and that $\varphi_{0} \in C^{1, \mu}\left(\mathbf{R}^{2}\right)$. In view of (2.3) the vorticity $\omega(x)=\partial_{1} v_{2}(x)-\partial_{2} v_{1}(x)$ is

$$
\omega(x, t)=\omega_{0} \chi_{D(t)}(x)
$$

with $\chi_{D}(x)=1$ if $x \in D, \frac{1}{2}$ if $x \in \partial D$ and 0 if $x \notin \bar{D}$.

It is well known that the Euler equations possess unique weak solutions whose vorticity obeys (2.7) ([10]). This velocity field is quasi-Lipschitz continuous; particle trajectories are unique, globally defined for $(\alpha, t) \in \mathbf{R}^{2} \times \mathbf{R}$ and Hölder continuous in both space and time. This classical Yudovich solution provides a solution of $(2.1-2.2)$ via

$$
\varphi(x, t)=\varphi_{0}\left(X^{-t}(x, t)\right) .
$$

The area of $D(t)$ is constant in time and is used to define the units of length. We set

$$
L^{2}=\operatorname{area}\left(D_{0}\right)=\operatorname{area}(D(t)) .
$$

We also use the notation

$$
\begin{aligned}
|\nabla \varphi(\cdot)|_{\text {inf }} & =\inf _{x \in \partial D}|\nabla \varphi(x)|=\inf _{x, \varphi(x)=0}|\nabla \varphi(x)|, \\
|\nabla \varphi(\cdot)|_{\mu} & =\sup _{x \neq x^{\prime}} \frac{\left|\nabla \varphi(x)-\nabla \varphi\left(x^{\prime}\right)\right|}{\left|x-x^{\prime}\right|^{\mu}}, \quad 0<\mu<1 .
\end{aligned}
$$

The main result is one of regularity of $\varphi$ :

Theorem. Given $\omega_{0} \neq 0, D_{0}$ bounded and $\varphi_{0} \in C^{1, \mu}\left(\mathbf{R}^{2}\right)$ satisfying (2.5) and (2.6) there exists a constant $C$ depending only on $\left|\omega_{0}\right|, L,\left|\nabla \varphi_{0}\right|_{\mu},\left|\nabla \varphi_{0}\right|_{L^{\infty}}$, and $\left|\nabla \varphi_{0}\right|_{\text {inf }}$ so that the problem (2.1)-(2.5) has a unique solution $\varphi(x, t)$ defined for all $x \in \mathbf{R}^{2}$ and $t \in \mathbf{R}$ and satisfying

$$
\begin{aligned}
|\nabla v(\cdot, t)|_{L^{\infty}} & \leqq|\nabla v(\cdot, 0)|_{L^{\infty}} e^{C|t|}, \\
|\nabla \varphi(\cdot, t)|_{\mu} & \leqq|\nabla \varphi(\cdot, 0)|_{\mu} \exp \left(\left(C_{0}+\mu\right) e^{C|t|}\right), \\
|\nabla \varphi(\cdot, t)|_{L^{\infty}} & \leqq|\nabla \varphi(\cdot, 0)|_{L^{\infty}} \exp \left(e^{C|t|}\right), \\
|\nabla \varphi(\cdot, t)|_{\text {inf }} & \leqq|\nabla \varphi(\cdot, 0)|_{\text {inf }} \exp \left(-e^{C|t|}\right) .
\end{aligned}
$$

$C_{0}$ is constant depending only on the dimension of the space. It is known [2] that (2.10)-(2.13) are sufficient for higher regularity. More precisely, the following theorem holds

Theorem. Consider $\omega_{0} \neq 0, D_{0}$ bounded and $\varphi_{0} \in C^{k, \mu}\left(\mathbf{R}^{2}\right)$ satisfying (2.5) and (2.6). Let $\varphi(x, t)$ be as defined in (2.8). A sufficient condition for $\varphi(x, t) \in C^{k, \mu}\left(\mathbf{R}^{2}\right)$ for all $t \in[0, T]$ is that $|\nabla \varphi(\cdot, t)|_{\mu},|\nabla \varphi(\cdot, t)|_{L^{\infty}}$, and $1 /\left(|\nabla \varphi(\cdot, t)|_{\text {inf }}\right)$ remain bounded for all $t \in[0, T]$.

In particular the above theorem is true for $C^{\infty}=\bigcap_{k=1}^{\infty} C^{k, \mu}$. We start with a few kinematic results.

Proposition 1. Assume $v$ is given by the Biot-Savart formula (2.3) and $\varphi$ is related to $D$ by (2.5). Then

$$
|\nabla v|_{L^{\infty}} \leqq\left(\frac{9}{2}+\frac{2}{\mu}\right)\left|\omega_{0}\right|\left(1+\left(\log \left[\Delta_{\mu}\right]\right)\right)
$$


where

$$
\Delta_{\mu}=\frac{|\nabla \varphi(\cdot)|_{\mu} L^{\mu}}{|\nabla \varphi(\cdot)|_{\mathrm{inf}}}
$$

In view of (1.3) we only need to estimate the symmetric part of $(\nabla v)\left(x_{0}\right)$ and the only nontrivial case is when $x_{0}$ is close to the boundary of $D$. Let us denote

$$
d\left(x_{0}\right)=\inf _{x \in \partial D}\left\{\left|x-x_{0}\right|\right\}
$$

the distance from $x_{0}$ to $\partial D$. We take a cutoff distance $0<\delta \leqq \infty$ defined by

$$
\delta^{\mu}=\frac{|\nabla \varphi|_{\text {inf }}}{|\nabla \varphi|_{\mu}}
$$

and consider the set of points $x_{0}$ so that

$$
d\left(x_{0}\right)<\delta .
$$

For every $\rho, \rho \geqq d\left(x_{0}\right)$, consider the directions $z$ so that $x_{0}+\rho z \in D$ :

$$
S_{\rho}\left(x_{0}\right)=\left\{z|| z \mid=1, x_{0}+\rho z \in D\right\} .
$$

Also, choose a point $\tilde{x} \in \partial D$ so that $\left|x_{0}-\tilde{x}\right|=d\left(x_{0}\right)$ and consider the semicircle

$$
\Sigma\left(x_{0}\right)=\left\{z|| z \mid=1, \nabla_{x} \varphi(\tilde{x}) \cdot z \geqq 0\right\} .
$$

As $d\left(x_{0}\right)$ approaches zero the symmetric difference

$$
R_{\rho}\left(x_{0}\right)=\left(S_{\rho}\left(x_{0}\right) \backslash \Sigma\left(x_{0}\right)\right) \cup\left(\Sigma\left(x_{0}\right) \backslash S_{\rho}\left(x_{0}\right)\right)
$$

becomes negligible. More precisely we have the following

Geometric Lemma. If $R_{\rho}\left(x_{0}\right)$ is the symmetric difference in (2.20) and $H^{1}$ denotes the Lebesgue measure on the unit circle then

$$
H^{1}\left(R_{\rho}\left(x_{0}\right)\right) \leqq 2 \pi\left(\left(1+2^{\mu}\right) \frac{d\left(x_{0}\right)}{\rho}+2^{\mu}\left(\frac{\rho}{\delta}\right)^{\mu}\right)
$$

holds for all $\rho \geqq d\left(x_{0}\right), \mu>0$ and $x_{0}$ so that $d\left(x_{0}\right)<\delta=\left(\frac{|\nabla \varphi|_{\text {inf }}}{|\nabla \varphi|_{\mu}}\right)^{1 / \mu}$.

The proof of this lemma is elementary and can be found in the appendix.

Proof of Proposition 1. Recall that we only need an estimate for the symmetric part of $\nabla v$ which we write as the sum of two integrals $I_{1}$ and $I_{2} . I_{2}$ is the integral

$$
I_{2}\left(x_{0}\right)=\frac{\omega_{0}}{2 \pi} \int_{D \cap\left\{\left|x_{0}-y\right| \geqq \delta\right\}} \frac{\sigma\left(x_{0}-y\right)}{\left|x_{0}-y\right|^{2}} d y,
$$

where $\delta$ is defined in (2.16). It follows that

$$
\left|I_{2}\right| \leqq\left|\omega_{0}\right|\left(1+\log \left(\frac{\delta}{L}\right)\right) .
$$


On the other hand

$$
I_{1}\left(x_{0}\right)=\frac{\omega_{0}}{2 \pi} \int_{D \cap\left\{\left|x_{0}-y\right|<\delta\right\}} \frac{\sigma\left(x_{0}-y\right)}{\left|x_{0}-y\right|^{2}} d y
$$

vanishes if $d\left(x_{0}\right)>\delta$ so we may assume that $d\left(x_{0}\right) \leqq \delta$. Passing to polar coordinates centered at $x_{0}$ and using $\int_{\Sigma\left(x_{0}\right)} \sigma d H^{1}(z)=0$ we obtain

$$
\left|I_{1}\left(x_{0}\right)\right| \leqq \frac{\left|\omega_{0}\right|}{2 \pi} \int_{d\left(x_{0}\right)}^{\delta} \frac{d \rho}{\rho} H^{1}\left(R_{\rho}\left(x_{0}\right)\right) .
$$

Applying the Geometric Lemma and integrating yields

$$
\left|I_{1}\right| \leqq\left|\omega_{0}\right|\left[\left(1+2^{\mu}\right)+\frac{2^{\mu}}{\mu}\right]
$$

which finishes the proof.

Proposition 2. If $v$ is given by the Biot-Savart formula (2.3) and $\mathbf{W}$ is a divergence free vector field tangent to $\partial D$ then

$$
\nabla v(x) \mathbf{W}=\frac{\omega_{0}}{2 \pi} P_{v} \int_{D} \frac{\sigma(x-y)}{|x-y|^{2}}(\mathbf{W}(x)-\mathbf{W}(y)) d y .
$$

The proof is a calculation based on (1.3):

$$
\begin{aligned}
& \frac{1}{2 \pi} P_{v} \int_{D} \nabla\left[\nabla_{y}^{\perp} \log |x-y|\right] \mathbf{W}(y) d y \\
& \quad=-\lim _{\delta \rightarrow 0} \frac{\omega_{0}}{2 \pi} \int_{|x-y|=\delta, y \in D}\left(\mathbf{W}(y) \cdot\left(\frac{x-y}{\delta}\right)\right) \nabla_{y}^{\perp} \log |x-y| d y \\
& \quad=-\frac{1}{2} \chi_{D}(x)\left(\begin{array}{rr}
0 & -1 \\
1 & 0
\end{array}\right) \mathbf{W}(x) .
\end{aligned}
$$

The first equality is due to the fact that $\mathbf{W}$ is divergence free and tangent to $\partial D$. We also use the fact that $\nabla_{y}\left[\nabla_{y}^{\perp} \log |x-y|\right]=\frac{\sigma(x-y)}{|x-y|^{2}}$. A corollary to Proposition 2 is

Corollary 1. There exists a constant $C_{0}$ such that if $v$ is given by the Biot-Savart formula (2.3) and if $\mathbf{W}$ is a divergence free vector field $\mathbf{W} \in C^{\mu}\left(\mathbf{R}^{2}, \mathbf{R}^{2}\right)$ tangent to $\partial D$ then

$$
|\nabla v \mathbf{W}|_{\mu} \leqq C_{0}|\nabla v|_{L^{\infty}}|\mathbf{W}|_{\mu} .
$$

The proof is straightforward and is given in the appendix.

Finally, here is the only time dependent result we need to conclude:

Proposition 3. Assume $\varphi$ is a solution of (2.1)-(2.5) on some interval of time $|t|<T$. Assume that $\varphi(\cdot, t) \in C^{1, \mu}\left(\mathbf{R}^{2}\right)$ and that $|\nabla \varphi(\cdot, t)|_{\text {inf }}>0$ for $|t| \leqq T$. Then

$$
|\nabla \varphi(\cdot, t)|_{\mu} \leqq|\nabla \varphi(\cdot, 0)|_{\mu} \exp \left[\left(C_{0}+\mu\right) \int_{0}^{t}|\nabla v(\cdot, s)|_{L^{\infty}} d s\right],
$$




$$
\begin{aligned}
& |\nabla \varphi(\cdot, t)|_{L^{\infty}} \leqq|\nabla \varphi(\cdot, 0)|_{L^{\infty}} \exp \left[\int_{0}^{t}|\nabla v(\cdot, s)|_{L^{\infty}} d s\right], \\
& |\nabla \varphi(\cdot, t)|_{\text {inf }} \geqq|\nabla \varphi(\cdot, 0)|_{\text {inf }} \exp \left[-\int_{0}^{t}|\nabla v(\cdot, s)|_{L^{\infty}} d s\right] .
\end{aligned}
$$

The proof of Proposition 3 is straightforward and is found in the appendix. It uses the corollary only for (2.24).

One concludes using Proposition 1 in conjunction with Proposition 3 that the estimates in the theorem are true as long as one has a solution. But local in time existence and uniqueness under the hypothesis of the theorem are easily obtained (see for example [2]). This finishes the proof of the theorem.

Remark. Using the same approach one can prove that several disjoint patches retain the smoothness of their boundaries. Also, under appropriate assumptions the result holds for a patch of nonconstant vorticity.

\section{Appendix}

Proof of the Geometric Lemma:

Recall that

$$
\begin{aligned}
S_{\rho}\left(x_{0}\right) & =\left\{z|| z \mid=1, x=x_{0}+\rho z \in D\right\}, \\
\Sigma\left(x_{0}\right) & =\left\{z|| z \mid=1\left(\nabla_{x} \varphi(\tilde{x}) \cdot(z)\right) \geqq 0\right\}, \\
R_{\rho}\left(x_{0}\right) & =\left(S_{\rho}\left(x_{0}\right) \backslash \Sigma\left(x_{0}\right)\right) \cup\left(\Sigma\left(x_{0}\right) \backslash S_{\rho}\left(x_{0}\right)\right) .
\end{aligned}
$$

Let $\theta(z)$ be the angle in $R_{\rho}\left(x_{0}\right)$ that corresponds to the point $z$ in $R_{\rho}\left(x_{0}\right)$. Parametrize $R_{\rho}$ by $\theta(z)$ given by

$$
\sin \theta(z)=\frac{\nabla \varphi(\tilde{x}) \cdot(z)}{|\nabla \varphi(\tilde{x})||z|}=\frac{\nabla \varphi(\tilde{x}) \cdot\left(\tilde{x}-x_{0}\right)}{|\nabla \varphi(\tilde{x})| \rho}+\frac{\nabla \varphi(\tilde{x}) \cdot\left(x_{0}+\rho z-\tilde{x}\right)}{|\nabla \varphi(\tilde{x})| \rho} .
$$

If $z \in R_{\rho}\left(x_{0}\right)$ the either $\left\{\sin \theta(z)>0\right.$ and $\left.\varphi\left(x_{0}+\rho z\right)<0\right\}$ or $\{\sin \theta(z)<0$ and $\left.\varphi\left(x_{0}+\rho z\right)>0\right\}$. In either case, since $\varphi(\tilde{x})=0$ and $\nabla \varphi(\tilde{x})$ is parallel to $x_{0}-\tilde{x}$,

$$
\begin{aligned}
|\sin \theta(z)| & \leqq \frac{d\left(x_{0}\right)}{\rho}+\left|\frac{\nabla \varphi(\tilde{x}) \cdot\left(x_{0}+\rho z-\tilde{x}\right)}{|\nabla \varphi(\tilde{x})| \rho}-\frac{\varphi\left(x_{0}+\rho z\right)-\varphi(\tilde{x})}{|\nabla \varphi(\tilde{x})| \rho}\right| \\
& \leqq \frac{d\left(x_{0}\right)}{\rho}+\frac{|\nabla \varphi|_{\mu}\left|x_{0}+\rho z-\tilde{x}\right|^{1+\mu}}{\rho|\nabla \varphi|_{\text {inf }}} \\
& \leqq \frac{d\left(x_{0}\right)}{\rho}+\frac{|\nabla \varphi|_{\mu}}{\rho|\nabla \varphi|_{\text {inf }}}\left(d\left(x_{0}\right)+\rho\right)^{1+\mu} \\
& \leqq \frac{d\left(x_{0}\right)}{\rho}+2^{\mu} \frac{|\nabla \varphi|_{\mu}}{\rho|\nabla \varphi|_{\text {inf }}}\left(d\left(x_{0}\right)^{1+\mu}+\rho^{1+\mu}\right) .
\end{aligned}
$$


Hence

$$
\begin{aligned}
H^{1}\left(R \rho\left(x_{0}\right)\right) & \leqq 2 \pi\left[\frac{d\left(x_{0}\right)}{\rho}+2^{\mu} \frac{|\nabla \varphi|_{\mu}}{\rho|\nabla \varphi|_{\text {inf }}}\left(d\left(x_{0}\right)^{1+\mu}+\rho^{1+\mu}\right)\right] \\
& \leqq 2 \pi\left[\left(1+2^{\mu}\right)\left(\frac{d\left(x_{0}\right)}{\rho}\right)+2^{\mu}\left(\frac{\rho}{\delta}\right)^{\mu}\right] .
\end{aligned}
$$

We prove the Corollary, in its general form:

Lemma. Let $K$ be a Calderon-Zygmund kernel, homogeneous of degree - n, with mean zero on spheres, satisfying $|\nabla K(x)| \leqq C|x|^{-n-1}$. There exists a constant $C_{0}$ so that all $f \in C^{\mu}\left(\mathbf{R}^{n}\right)$ and $\omega \in L^{\infty}\left(\mathbf{R}^{n}\right)$ satisfy

where

$$
|G|_{\mu} \leqq C_{0}(\mu, n)|f|_{\mu}\left(|K * \omega|_{L^{\infty}}+|\omega|_{L^{\infty}}\right),
$$

$$
G(x)=P_{v} \int_{\mathbf{R}^{n}} K(x-y)(f(x)-f(y)) \omega(y) d y .
$$

Proof. We write $G(x)-G(x+h)=$

$$
\begin{aligned}
& P_{v} \int K(x-y)(f(x)-f(y)) \omega(y) d y-P_{v} \int K(x+h-y)(f(x+h)-f(y)) \omega(y) d y \\
& =P_{v} \int_{|x-y|<2 h} K(x-y)(f(x)-f(y)) \omega(y) d y \\
& \quad-P_{v} \int_{|x-y|<2 h} K(x+h-y)(f(x+h)-f(y)) \omega(y) d y \\
& \quad+P_{v} \int_{|x-y| \geqq 2 h} K(x-y)(f(x)-f(x+h)) \omega(y) d y \\
& \quad+P_{v} \int_{|x-y| \geqq 2 h}[K(x-y)-K(x+h-y)](f(x+h)-f(y)) \omega(y) d y \\
& =(1)+(2)+(3)+(4) .
\end{aligned}
$$

Clearly $|(1)|,|(2)| \leqq C_{\mu}|f|_{\mu} h^{\mu}|\omega|_{L^{\infty}}$. Also, we have

$$
|(4)| \leqq \int_{|x-y| \geqq 2 h} h \frac{C}{|x-y|^{n+1-\mu}}|f|_{\mu}|\omega|_{L^{\infty}} d y \leqq C_{\mu} h^{\mu}|f|_{\mu}|\omega|_{L^{\infty}} .
$$

A bound for (3) is obtained by using a lemma due to Cotlar (see Torchinsky [9] p. 291):

$$
|(3)| \leqq|f|_{\mu} h^{\mu} \frac{1}{2 \pi}\left|P_{v} \int_{|x-y| \geqq 2 h} K(x-y) \omega(y) d y\right| \leqq C_{0}|f|_{\mu} h^{\mu}\left(|K * \omega|_{L^{\infty}}+|\omega|_{L^{\infty}}\right) .
$$

To prove Lemma 2 we recall that $\mathbf{W}=\nabla^{\perp} \varphi$ satisfies

$$
\frac{\partial \mathbf{W}}{\partial t}+v \cdot \nabla \mathbf{W}=\nabla v \mathbf{W}
$$

Let $X(\alpha, t)$ be the particle trajectory map associated with $v$. The bounds (2.25) and (2.26) can be obtained directly by writing (3.4) in Lagrangian coordinates and computing pointwise bounds for $|\mathbf{W}|$. That is,

$$
\mathbf{W}(X(\alpha, t), t)=\mathbf{Z}(\alpha, t)
$$


so that $\mathbf{Z}$ satisfies

$$
\begin{gathered}
\frac{d \mathbf{Z}(\alpha, t)}{d t}=\nabla v(X(\alpha, t), t) \mathbf{Z}(\alpha, t), \\
\frac{d \ln |\mathbf{Z}(\alpha, t)|}{d t} \leqq|\nabla v(X(\alpha, t), t)|, \\
e^{-\int_{0}^{t}|\nabla v(\cdot, s)| L^{\infty} d s} \leqq \frac{|\mathbf{Z}(\alpha, t)|}{|\mathbf{Z}(\alpha, 0)|} \leqq e^{-\int_{0}^{t}|\nabla v(\cdot, s)| L^{\alpha} d s},
\end{gathered}
$$

which certainly implies (2.25) and (2.26).

We present the details of (2.24). We write

$$
\mathbf{W}(x, t)=\mathbf{W}_{0}\left(X^{-1}(x, t)\right)+\int_{0}^{t} \nabla v \mathbf{W}\left(X^{-1}(x, t-s), s\right) d s .
$$

So that $\left|\mathbf{W}(x, t)-\mathbf{W}\left(x^{\prime}, t\right)\right| \leqq$

$$
\begin{aligned}
& \left|\mathbf{W}_{0}\left(X^{-1}(x, t)\right)-\mathbf{W}_{0}\left(X^{-1}\left(x^{\prime}, t\right)\right)\right| \\
& \quad+\left|\int_{0}^{t} \nabla v \mathbf{W}\left(X^{-1}(x, t-s), s\right)-\nabla v \mathbf{W}\left(X^{-1}\left(x^{\prime}, t-s\right), s\right) d s\right| \\
& \leqq \\
& \qquad\left.\mathbf{W}_{0}\right|_{\mu}\left|\nabla X^{-1}(\cdot, t)\right|_{L^{\infty}}^{\mu}\left|x-x^{\prime}\right|^{\mu}+\int_{0}^{t}|\nabla v \mathbf{W}(\cdot, s)|_{\mu}\left|\nabla X^{-1}(\cdot, t-s)\right|_{L^{\infty}}^{\mu}\left|x-x^{\prime}\right|^{\mu} d s \\
& \leqq\left|\mathbf{W}_{0}\right|_{\mu} \exp \left[\mu \int_{0}^{t}|\nabla v(\cdot, s)|_{L^{\infty}} d s\right]\left|x-x^{\prime}\right|^{\mu} \\
& \quad+\int_{0}^{t}|\nabla v \mathbf{W}(\cdot, s)|_{\mu} \exp \left[\mu \int_{s}^{t}\left|\nabla v\left(s^{\prime}, \cdot\right)\right|_{L^{\infty}} d s^{\prime}\right]\left|x-x^{\prime}\right|^{\mu} d s
\end{aligned}
$$

and therefore

$$
\begin{aligned}
|\mathbf{W}(\cdot, t)|_{\mu} \leqq & \left|\mathbf{W}_{0}\right|_{\mu} \exp \left[\mu \int_{0}^{t}|\nabla v(\cdot, s)|_{L^{\infty}} d s\right] \\
& +\int_{0}^{t}|\nabla v \mathbf{W}(\cdot, s)|_{\mu} \exp \left[\mu \int_{s}^{t}\left|\nabla v\left(\cdot, s^{\prime}\right)\right|_{L^{\infty}} d s^{\prime}\right] d s .
\end{aligned}
$$

Here we use the fact that $\nabla_{x} X^{-1}$ satisfies

$$
\left|\nabla X^{-1}(x, t-s)\right|_{L^{\infty}} \leqq \exp \left[\int_{s}^{t}\left|\nabla v\left(s^{\prime}, \cdot\right)\right|_{L^{\infty}} d s^{\prime}\right] .
$$

Writing $Q(s)=|\nabla v(\cdot, s)|_{L^{\infty}}$, and using (2.23) we have

$$
|\mathbf{W}(\cdot, t)|_{\mu} \leqq\left|\mathbf{W}_{0}\right|_{\mu} \exp \left[\mu \int_{0}^{t} Q(s) d s\right]+C \int_{0}^{t} Q(s)|\mathbf{W}(\cdot, s)|_{\mu} \exp \left[\mu \int_{s}^{t} Q\left(s^{\prime}\right) d s^{\prime}\right] d s .
$$

Multiplying both sides by $\exp \left[-\mu \int_{0}^{t} Q\left(s^{\prime}\right) d s^{\prime}\right]$ we obtain

$$
|\mathbf{W}(\cdot, t)|_{\mu} \exp \left[-\mu \int_{0}^{t} Q\left(s^{\prime}\right) d s^{\prime}\right] \leqq\left|\mathbf{W}_{0}\right|_{\mu}+C_{0} \int_{0}^{t} Q(s)|\mathbf{W}(\cdot, s)|_{\mu} \exp \left[-\mu \int_{0}^{s} Q\left(s^{\prime}\right) d s^{\prime}\right] d s \text {. }
$$


So that $|\mathbf{W}(\cdot, t)|_{\mu} \exp \left[-\mu \int_{0}^{t} Q\left(s^{\prime}\right) d s^{\prime}\right]=G(t)$ satisfies

$$
G(t) \leqq\left|\mathbf{W}_{0}\right|_{\mu}+C_{0} \int_{0}^{t} Q(s) G(s) d s,
$$

and thus by Gronwall's Lemma satisfies

$$
G(t) \leqq\left|\mathbf{W}_{0}\right|_{\mu} \exp \left[C_{0} \int_{0}^{t} Q(s) d s\right]
$$

which gives

$$
|\mathbf{W}(\cdot, t)|_{\mu} \leqq\left|\mathbf{W}_{0}\right|_{\mu} \exp \left[\left(C_{0}+\mu\right) \int_{0}^{t}|\nabla v(\cdot, s)|_{L^{\infty}} d s\right]
$$

\section{References}

1. Alinhac, S.: Remarques sur l'instabilité du problème des poches de tourbillon. Prépublication de l'Université d'Orsay, à paraitre dans J. Funct. Anal. 1989.

2. Bertozzi, A.: Existence, Uniqueness, and a Characterization of Solutions to the Contour Dynamics Equation. PhD thesis, Princeton University 1991

3. Buttke, T.F.: The observation of singularities in the boundary of patches of constant vorticity. Phys. Fluids A 1, 1283-1285 (1989)

4. Chemin, J.-Y.: Persistance de structures geometriques dans les fluides incompressibles bidimensionnels. Preprint 1991

5. Constantin, P., Lax, P.D., Majda, A.: A simple one dimensional model for the three dimensional vorticity equation. Commun. Pure Applied Math. 38, 715-724 (1985)

6. Constantin, P. and Titi, E.S.: On the evolution of nearly circular vortex patches. Commun. Math. Phys. 119, 177-198 (1988)

7. Dritschel, D.G., McIntyre, M.E.: Does contour dynamics go singular? Phys. Fluids A, 2(5) 748-753 (1990)

8. Majda, A.: Vorticity and the mathematical theory of incompressible fluid flow. Commun. Pure Appl. Math. 39, 5187-5220 (1986)

9. Torchinsky, A.: Real Variable Methods in Harmonic Analysis. New York: Academic Press 1986

10. Yudovich, V.I.: Non-stationary flow of an ideal incompressible liquid. Zh. Vych. Mat. 3, 1032-1066 (1963) (in Russian)

11. Zabusky, N., Hughes, M.H., Roberts, K.V.: Contour dynamics for the Euler equations in two dimensions. J. Comp. Phys. 96-106 (1979)

Communicated by A. Jaffe 